\title{
TWISTING OPERATIONS AND COMPOSITE KNOTS
}

\author{
MASAKAZU TERAGAITO
}

(Communicated by Ronald Stern)

\begin{abstract}
Suppose that a composite knot $K$ in $S^{3}$ can be changed to a trivial knot by $1 / n$-surgery along a trivial loop $C$. We show that $|n| \leq 2$. Moreover, if there is a decomposing sphere of $K$ which meets $C$ in two points, then $|n| \leq 1$.
\end{abstract}

\section{INTRODUCTION}

Let $K$ be a knot in the 3-sphere $S^{3}$ and $D$ a disk which intersects $K$ transversely in its interior. Let $C=\partial D$. We get a new knot $K^{*}$ in $S^{3}$ as the image of $K$ after doing $1 / n$-surgery along $C$. We say that $K^{*}$ is obtained from $K$ by $n$-twisting along $C$. In particular, this operation is called a trivializing $n$ twist of $K$ if $K^{*}$ is unknotted. We remark that a crossing change is equivalent to \pm 1 -twist on a disk which intersects $K$ in precisely two points.

In [4], Mathieu asked if there is a composite knot which admits a trivializing twist. Several families of composite knots are known to admit trivializing twists at present [5], [7], [11]. Since all the examples of trivializing twists of composite knots are \pm 1 -twists, it is conjectured that if a composite knot admits a trivializing $n$-twist, then $|n| \leq 1$ [6]. In fact, Motegi [6] proved that $|n| \leq 5$, by making use of Gordon's result about Dehn fillings on hyperbolic manifolds [2].

In this paper we improve Motegi's result as follows.

Theorem 1. If a composite knot admits a trivializing $n$-twist, then $|n| \leq 2$.

The possibility of $|n|=2$ remains an open problem.

If a knot $K$ is composite, then there is a 2-sphere $S$ which intersects $K$ transversely in two points, such that each one of the 3-balls bounded by $S$ intersects $K$ in a knotted spanning arc. Such a sphere is called a decomposing sphere of $K$.

Theorem 2. Suppose that a composite knot $K$ admits a trivializing $n$-twist along $C$ and that there is a decomposing sphere $S$ of $K$ which intersects $C$ transversely in two points. Then $|n| \leq 1$.

Received by the editors September 7, 1993.

1991 Mathematics Subject Classification. Primary 57M25.

Key words and phrases. Knot, twisting. 


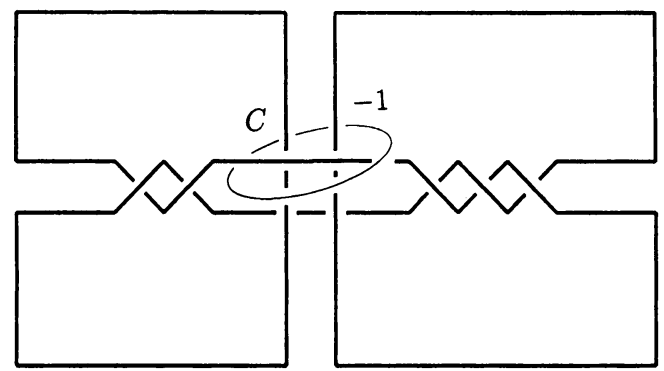

$K$

twisting

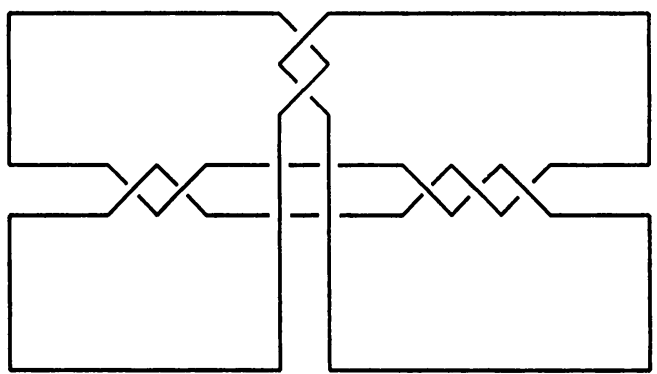

$K^{*}$ : unknot

Figure 1

It is easy to verify that all known examples in the above papers satisfy this assumption. An example is illustrated in Figure 1.

Scharlemann [9] proved that unknotting number one knots are prime. That is, a composite knot cannot be trivialized by \pm 1 -twists on a disk which meets the knot in two points. (See also [10].) Miyazaki-Yasuhara [5] found many examples of composite knots which do not admit trivializing twists.

I would like to thank the referee for helpful comments.

\section{Preliminaries}

Let $K$ be a composite knot in $S^{3}$. Suppose that $K$ admits a trivializing $n$-twist along $C$. Let $M=S^{3}-$ Int $N(K \cup C)$. Let us write $T=\partial N(C), T^{\prime}=$ $\partial N(K)$. Slopes on $T$ or $T^{\prime}$ will be parametrized by $\mathscr{Q} \cup\{1 / 0\}$ in the usual way (cf. [8]), using a meridian-longitude basis. Since $K \cup C$ is unsplittable in $S^{3}, M$ is irreducible. For any slope $r$ on $T$, let $M(r)$ denote the manifold obtained from $M$ by $r$-Dehn filling on $T$, that is, by attaching a solid torus $J$ to $M$ along $T$ so that $r$ bounds a disk in $J$. It is immediate from the definitions that $K$ is trivialized by $n$-twisting along $C$ if and only if $M(1 / n)$ is a solid torus. Note that $M(1 / 0)=S^{3}-\operatorname{Int} N(K)$.

Let $S$ be a decomposing sphere of $K$. Isotope $S$ so that $S \cap N(C)$ is a disjoint union of meridian disks of $N(C)$ and $m=|S \cap N(C)|$ is minimal. Note that $m \geq 2$. Then $P=S \cap M$ is an incompressible planar surface in $M$, with two outer boundary components $\partial_{0} P, \partial_{\infty} P$, lying in $T^{\prime}$, and $m$ inner boundary components $\partial_{i} P, i=1, \ldots, m$, lying in $T$. Here, the inner 
boundary components are numbered so that they are consecutive on $T$. Each component of $\partial P$ has slope $1 / 0$ in $T$ or $T^{\prime}$.

Let $D_{0}$ be a meridian disk of $M(1 / n)$. Isotope $D_{0}$ so that $D_{0} \cap J$ is a disjoint union of meridian disks of $J$. We choose $D_{0}$ so that $l=\left|D_{0} \cap J\right|$ is minimal over all meridian disks of $M(1 / n)$. Note that $l \geq 2$. If $l=1$, then we regard $J$ as a regular neighborhood of a core of $M(1 / n)$. This would imply that $M(1 / 0)$ is a solid torus. From the minimality of $l, Q=D_{0} \cap M$ is an incompressible planar surface in $M$, with one outer boundary component $\partial_{0} Q$, lying in $T^{\prime}$, and $l$ inner boundary components $\partial_{j} Q, j=1, \ldots, l$, each having slope $1 / n$ in $T$. The inner boundary components are numbered consecutively on $T$. It is easy to see that $\partial_{0} Q$ has slope $n \omega^{2} / 1$, where $\omega=l k(K, C)$.

By an isotopy of $Q$, we may assume that $P$ and $Q$ intersect transversely, and each outer boundary component of $P$ intersects $\partial_{0} Q$ exactly once, and each inner boundary component of $P$ intersects each inner boundary of $Q$ in $|n|$ points. Thus, for example, when we go around an inner boundary component of $P$, we will consecutively meet $\partial_{1} Q, \partial_{2} Q, \ldots, \partial_{l} Q, \ldots, \partial_{1} Q, \ldots, \partial_{l} Q$ (repeated $|n|$ times). By an innermost argument, we can assume that no loop component of $P \cap Q$ bounds a disk in $P$ or $Q$, since $P$ and $Q$ are incompressible and $M$ is irreducible.

As in [1], we form the associated graphs $G_{P}$ and $G_{Q}$. Let $A$ be the annulus obtained by capping off the inner boundary components of $P$ by meridian disks of $N(C)$. We obtain a graph $G_{P}$ in $A$ by taking as the "fat" vertices of $G_{P}$ the disks in $N(C)$ that cap off the inner boundary components of $P$, and as the edges of $G_{P}$ the arc components of $P \cap Q$ in $P$. Similarly we obtain the graph $G_{Q}$ in the disk $D_{0}$.

Let $G$ denote either $G_{P}$ or $G_{Q}$.

If an edge $e$ connects a vertex to a vertex, then $e$ is an interior edge; otherwise, it is a boundary edge. Note that $G$ has at most two boundary edges. If $G_{P}$ has two boundary edges, so does $G_{Q}$, and vice versa. Each vertex of $G_{P}$ $\left(G_{Q}\right)$ has degree $|n| l \quad(|n| m$, resp. $)$.

Let $e$ be an edge of $G_{P}$. If an end point of $e$ is in $\partial_{i} P \cap \partial_{j} Q$, then we give this end point of $e$ the label $j$. Thus each incidence of an edge of $G_{P}$ at a vertex of $G_{P}$ is labeled with a vertex of $G_{Q}$. Similarly in $G_{Q}$, label the end points of edges incident to vertices.

Two vertices of $G_{P}\left(G_{Q}\right)$ are parallel if the corresponding inner boundary components of $P(Q)$, when given the orientations induced by some orientation of $P(Q)$, are homologous in $T$; otherwise, they are antiparallel. Since $M$ is orientable, we have the parity rule:

An interior edge $e$ of $G_{P}$ connects parallel vertices in $G_{P}$ if and only if $e$ connects antiparallel vertices in $G_{Q}$.

An $x$-cycle in $G$ is a cycle $\sigma$ of edges in $G$ such that all the vertices of $G$ in $\sigma$ are parallel and $\sigma$ can be oriented so that the tail of each edge has label $x$. A Scharlemann cycle in $G$ is an $x$-cycle $\sigma$ in $G$ for some label $x$ such that $\sigma$ bounds a disk face of $G$. In particular, a Scharlemann cycle of length 1 will be called a trivial loop.

Lemma 1. $G$ contains no trivial loops.

Proof. This follows immediately from the minimality of $l$ or $\mathrm{m}$.

Lemma 2. $G$ contains no Scharlemann cycles. 
The proof is analogous to [1, proof of Lemma 2.5.2] or [3, proof of Lemma 3.3]. We omit the details.

\section{ProOFs}

To find Scharlemann cycles, we consider the following conditions as in [1]:

(*) There exists a vertex $x$ of $G$ such that for each label $y$ there is an edge of $G$ incident to $x$ with label $y$, connecting $x$ to an antiparallel vertex of $G$.

(**) For each vertex $x$ of $G$ there exists a label $y(x)$ such that each edge of $G$ incident to $x$ with label $y(x)$ connects $x$ either to a parallel vertex of $G$ or to an outer boundary.

In fact, $(* *)$ is the negation of $(*)$.

Lemma 3. Suppose that $G_{P}$ satisfies (*). Then $G_{Q}$ contains a Scharlemann cycle.

Proof. See [1, Lemmas 2.6.2 and 2.6.3].

Remark. In general, we cannot exchange the roles of $P$ and $Q$ in the statement of Lemma 3. Because an $x$-cycle in $G_{P}$ does not necessarily bound a disk in the annulus $A$. However, when $G_{P}$ has only one boundary edge, we can conclude that $G_{P}$ contains a Scharlemann cycle if $G_{Q}$ satisfies $(*)$.

Lemma 4. Let $x$ be a vertex of $G_{P}$. If there exist successive $l$ edges of $G_{P}$ connecting $x$ to antiparallel vertices, then $G_{Q}$ contains a Scharlemann cycle.

Proof. This follows immediately from Lemma 3.

Lemma 5. If $G$ contains a parallel family of edges connecting parallel vertices, then either the sets of labels at the two ends of the family are disjoint, or $G$ contains a Scharlemann cycle. In particular, if $G_{P}\left(G_{Q}\right)$ contains a parallel family of more than $l / 2(\mathrm{~m} / 2$, resp.) edges connecting parallel vertices, then $G_{P}\left(G_{Q}\right)$ contains a Scharlemann cycle.

Proof. See [1, Lemma 2.6.6 and Corollary 2.6.7].

Lemma 6. Suppose that $|n| \geq 2$ and that $G_{Q}$ satisfies (**). Then either $G_{Q}$ contains a Scharlemann cycle or every vertex of $G_{P}$ belongs to a boundary edge of $G_{P}$.

Proof. This is essentially [1, Lemma 2.6.4]. The proof works well even if $G_{P}$ is a graph in an annulus.

We remark that the latter conclusion of Lemma 6 implies that $G_{P}$ has exactly two vertices.

Now suppose that $G_{P}$ satisfies $(* *)$. Let $v$ be a vertex of $G_{P}$. There exists a label $y(v)$ such that each one of $|n|$ edges of $G_{P}$ incident to $v$ with label $y(v)$ connects $v$ either to a parallel vertex or to $\partial A$. Fix the label $y(v)$. These $|n|$ edges will be called the $y(v)$-edges at $v$. A corner at $v$ is an interval on the boundary of the fat vertex $v$ between successive labels $y(v)$. There are $|n|$ corners around $v$, and there are $l-1$ incidences of edges to $v$ in the interior of a corner. Let $\Gamma=G_{P}-$ boundary edges $\}$. Let $\bar{\Gamma}$ be the reduced graph of $\Gamma$, obtained by amalgamating all mutually parallel edges in the obvious way. Then $G_{P}, \Gamma$, and $\bar{\Gamma}$ have the same vertex set.

We now want to estimate the degree $\operatorname{deg}_{\bar{\Gamma}}(v)$ of $v$ in $\bar{\Gamma}$. 
Lemma 7. Suppose that $G_{P}$ satisfies $(* *)$. Let $v$ be a vertex of $G_{P}$, and let $b(v)$ be the number of boundary edges incident to $v$. Then $\operatorname{deg}_{\bar{\Gamma}}(v) \geq 2|n|-b(v)$.

Proof. By Lemmas 2 and 5, any pair of $y(v)$-edges is not parallel. Hence the $y(v)$-edges, except for boundary edges, correspond to distinct edges of $\bar{\Gamma}$. Also, not all the $l-1$ edges incident to $v$ in the interior of a corner are parallel to $y(v)$-edges. Therefore, the interior of a corner yields at least one edge of $\bar{\Gamma}$ unless it does not meet a boundary edge. The conclusion follows from these observations.

In fact, we have three possibilities, according to the situation in $G_{P}$ :

(1) No boundary edge is incident to $v$.

(2) Only one boundary edge is incident to $v$.

(3) Two boundary edges are incident to $v$.

There is at most one vertex of $G_{P}$ that satisfies (3). If a vertex satisfies (2), then $G_{P}$ has precisely two such vertices.

The following lemma is an easy consequence of Lemma 7 and the observation above.

Lemma 8. Let $\Lambda$ be a component of $\bar{\Gamma}$. Let $V$ and $E$ be the number of vertices and edges of $\Lambda$. Then $|n| V \leq E+1$.

Possibly, $G_{P}$ is disconnected. Choose a point $z \in \partial A-G_{P}$. We define a partial ordering on the set of components of $G_{P}$ as in [1]. For two components $H_{1}$ and $H_{2}$ of $G_{P}, H_{1}<H_{2}$ if and only if every path in $A$ from $H_{1}$ to $z$ meets $H_{2}$ : A component of $G_{P}$ is extremal if it is minimal with respect to the partial ordering for some choice of $z$.

Proof of Theorem 1. Suppose that $|n| \geq 3$. If $G_{P}$ satisfies $(*)$, then $G_{Q}$ would contain a Scharlemann cycle by Lemma 3, contradicting Lemma 2. Thus $G_{P}$ satisfies $(* *)$.

We may assume that $G_{P}$ is connected. If $G_{P}$ is disconnected, we will replace $G_{P}$ by an extremal component. (We avoid a component without vertex.) We consider the reduced graph $\bar{\Gamma}$ of $\Gamma=G_{P}-\{$ boundary edge $\}$ as before. Since $G_{P}$ is connected, $\bar{\Gamma}$ is also connected. Let $V, E$, and $F$ be the number of vertices, edges, and faces of $\bar{\Gamma}$. We do not count the region meeting a component of $\partial A$ as a face of $\bar{\Gamma}$. By Lemma $8,3 V \leq E+1$. Since $\bar{\Gamma}$ has no 1-sided faces or parallel edges, every face has at least three sides. Let $F_{0}$ and $F_{\infty}$ be the components of $A-\bar{\Gamma}$ containing $\partial_{0} P$ and $\partial_{\infty} P$ respectively (possibly, $F_{0}=F_{\infty}$ ). The frontiers $\operatorname{Fr} F_{0}$ and $\operatorname{Fr} F_{\infty}$ can be expressed as the unions of a sequence of edges. Let $a$ and $b$ be the number of edges in $\operatorname{Fr} F_{0}$ and $\operatorname{Fr} F_{\infty}$ respectively. Note that a double edge is counted twice. Then $3 F+a \leq 2 E$ if $F_{0}=F_{\infty}$, or $3 F+(a+b) \leq 2 E$ if $F_{0} \neq F_{\infty}$. By Euler's formula, $1=V-E+F \leq \frac{1-a}{3}$ if $F_{0}=F_{\infty}$, or $0=V-E+F \leq \frac{1-(a+b)}{3}$ if $F_{0} \neq F_{\infty}$. This is a contradiction in either case. This completes the proof.

Proof of Theorem 2. Suppose that $|n| \geq 2$. Since $|S \cap N(C)|=2, G_{P}$ has exactly two vertices $x$ and $y$ that are antiparallel. If $G_{P}$ has only one boundary edge, then the arc corresponding to the boundary edge is essential in the annulus $A$. If $G_{Q}$ satisfies $(*)$, then $G_{P}$ contains a Scharlemann cycle by the remark after Lemma 3. If $G_{Q}$ satisfies $(* *)$, then Lemma 6 implies that $G_{Q}$ contains a Scharlemann cycle, since no vertex of $G_{P}$ belongs to a boundary edge. In either 


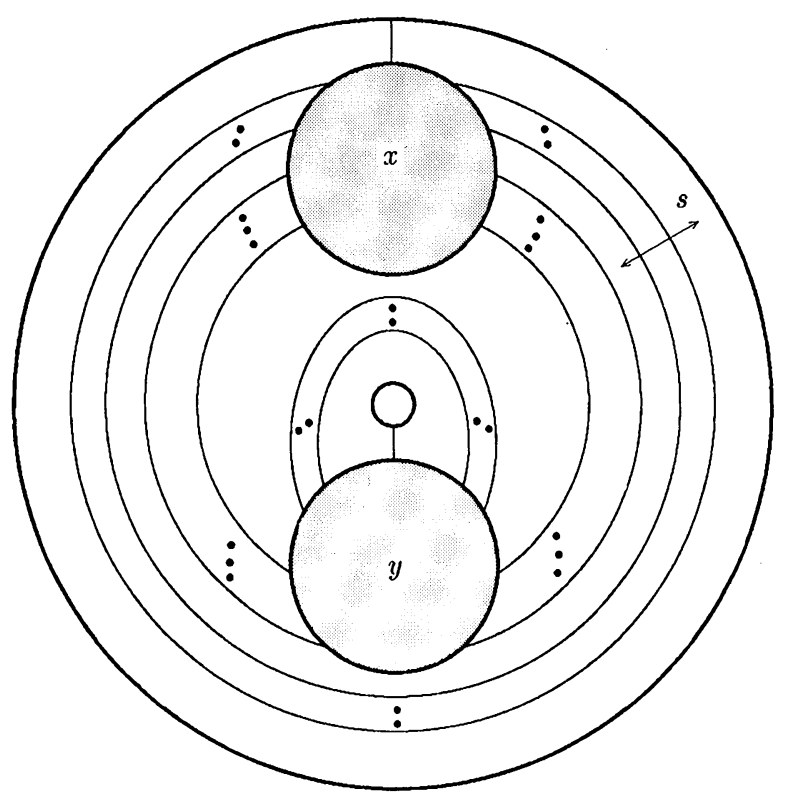

FIGURE 2

case, Lemma 2 gives a contradiction. Hence $G_{P}$ has exactly two boundary edges.

If two boundary edges are incident to the same vertex $x$, say, then there is a loop $\sigma$ at $y$, since $x$ and $y$ have the same degree. However, $\sigma$ bounds a disk which does not contain the vertex $x$. Hence, there would be a trivial loop. This contradicts Lemma 1 . Thus each vertex belongs to a single boundary edge.

We distinguish two cases.

(1) $G_{P}$ contains no loops.

Then all the interior edges incident to $x$ connect vertices $x$ and $y$. By Lemma $4, G_{Q}$ contains a Scharlemann cycle. This contradicts Lemma 2.

(2) $G_{P}$ contains a loop.

There is a loop based at $x$. Any loop must be essential in $A$. Consider the edge $e$ incident to $x$ immediately to the right of the boundary edge. Then $e$ must be a loop. Otherwise, a loop based at $x$ would be inessential in $A$. Then, without loss of generality, we have a situation as in Figure 2.

Suppose that there are $s$ parallel loops, including $e$. Then by Lemma 5 , $s \leq l / 2$. But if $s=l / 2$, then a loop has the same label at both ends, which contradicts the parity rule. Therefore, $2 s+1 \leq l$. Hence, there are at least $l$ edges connecting $x$ to $y$, since $x$ has degree $|n| l \geq 2 l$. Then, by Lemma 4, $G_{Q}$ contains a Scharlemann cycle, a contradiction. This completes the proof.

\section{REFERENCES}

1. M. Culler, C. McA. Gordon, J. Luecke, and P. B. Shalen, Dehn surgery on knots, Ann. of Math. (2) 125 (1987), 237-300.

2. C. McA. Gordon, When are tori created by Dehn surgery?, Conference Report for the International Conference on Knot Theory and Related Topics, 1990, pp. 18-19.

3. __ Combinatorial methods in knot theory, Algebra and Topology (S. H. Bae and G. T. 
Jin, eds.), Proc. KAIST Math. Workshop, vol. 5, 1990, pp. 1-23.

4. Y. Mathieu, Unknotting, knotting by twists on disks and property $(P)$ for knots in $S^{3}$, Knots 90 (A. Kawauchi, ed.), Proc. 1990 Osaka Conference on Knot Theory and Related Topics, de Gruyter, Berlin, 1992, pp. 93-102.

5. K. Miyazaki and A. Yasuhara, Knots that cannot be obtained from a trivial knot by twisting, preprint, 1993.

6. K. Motegi, Primeness of twisted knots, Proc. Amer. Math. Soc. 119 (1993), 979-983.

7. K. Motegi and T. Shibuya, Are knots obtained from a plain pattern prime?, Kobe J. Math. 9 (1992), 39-42.

8. D. Rolfsen, Knots and links, Math. Lecture Ser., vol. 7, Publish or Perish, Berkeley, CA, 1976.

9. M. Scharlemann, Unknotting number one knots are prime, Invent. Math. 82 (1985), 37-55.

10. M. Scharlemann and A. Thompson, Unknotting number, genus, and companion tori, Math. Ann. 280 (1988), 191-205.

11. M. Teragaito, Composite knots trivialized by twisting, J. Knot Theory Ramifications 1 (1992), 467-470.

Department of Mathematics, Kobe University, Rokko 1-1, NadA, Kobe 657, JaPan 